\title{
ULTRASOUND-GUIDED PUDENDAL NERVE BLOCK IN MALE DONKEYS
}

\author{
MOHAMED EL-SHERIF $^{1}$; AHMED EL-KHAMARY ${ }^{2}$; MOHAMED NAZIH ${ }^{3}$ and \\ WALEED SENOSY ${ }^{4}$ \\ ${ }^{1}$ Department of Surgery, Faculty of Veterinary Medicine, Assiut University, New Valley Branch, New Valley, Egypt. \\ ${ }^{2}$ Department of Surgery, Faculty of Veterinary Medicine, Damanhour University, Damanhour, Egypt. \\ ${ }^{3}$ Department of Anatomy, Faculty of Veterinary Medicine, Assiut University, New Valley Branch, New Valley, Egypt. \\ ${ }^{4}$ Department of Theriogenology, Faculty of Veterinary Medicine, Assiut University, New Valley branch, New Valley, \\ Egypt.
}

Received: 24 August 2017; $\quad$ Accepted: 24 October 2017

\begin{abstract}
The objective of this study was to develop a color flow doppler ultrasound-guided technique for the pudendal nerve block in male donkeys. A detailed anatomical dissection was performed in five donkey cadavers to obtain the precise details of pudendal nerve and its relations and ramifications, detect the pudendal nerve blood vessels and to determine the optimal point for local anesthetic injection. The pudendal nerve in six male native breed donkeys was blocked successfully with local anesthetic solution. The injection site was located with the aid of ultrasound. Ten milliliters of local analgesic solution were injected via 20-cm, 18-gauge needle. Anesthetic effect was evaluated after bilateral pudendal nerve blocks. A feasible, applicable and highly accurate technique to locate the pudendal nerve was developed. Satisfactory anesthesia of the anus, perineum, penis, prepuce and glans penis was achieved while the animal in a steady standing position.
\end{abstract}

Key words: Donkey, Nerve block, Pudendal nerve, Ultrasound-guided.

\section{INTRODUCTION}

Several surgical procedures in the equine male genital organs, often done under effect of epidural anesthesia or general anesthesia. Epidural anesthesia is not always an easy procedure in donkeys rather than horses, due to, anatomical variation. Moreover, epidural anesthesia has many drawbacks including; impairment of locomotor function, ataxia and recumbency during the procedure (Burnham 2002; Olbrich and Mosing, 2003; Natalini and Driessen, 2007).

General anesthesia has the disadvantage that it requires advanced tools and associated with high mortalities (Johnston et al., 2002). Regional anesthetic techniques were developed and reported to have a reliable anesthetic effect, a better pain relief and easier application. Pudendal nerve block is introduced as an efficient method to anesthetize anus, perineum and penis in male horses, in a steady standing position without impairment of locomotion function (Schumacher et al., 1985; Gallacher et al., 2016).

Corresponding author: Dr. MOHAMED EL-SHERIF

E-mail address:drmwt@ hotmail.com

Present address: Department of Surgery, Faculty of Veterinary Medicine, Assiut University, New Valley Branch, New Valley, Egypt.
Standard pudendal nerve block technique in horses was achieved by blind insertion of a long needle to the level of the nerve at the sacroisheatic ligament (Deshmkh and Deshpande, 1908; Schumacher et al., 1985). An alternative recent technique was introduced in horses by using a nerve-locating device to offer more accuracy for the procedure (Gallacher et al., 2016). Topographic anatomy of the pudendal nerve makes it hard to reach. The pudendal nerve originates as a common trunk with the caudal rectal nerve at the level of the second to third sacral vertebrae. It gives a superficial branch, ends at the caudal portion of sacrotubiral ligament and a deep branch, extended distally as nerve of the penis. Two to three centimeters cranial to the pudendal nerve, ischiatic nerve lies. The pudendal nerve is closely related to internal pudendal artery and vein (Ghoshal, 1975; Budras et al., 2012). Faulty introduction of the anesthetic needle may cause severe damage to the ischiatic nerve or bleeding from associated blood vessels. The purpose of this study was to develop a technique for ultrasound-guided pudendal nerve block in the male donkey. Also, the onset, duration and quality of anesthesia were recorded and evaluated.

\section{MATERIALS AND METHODS}

Topographical anatomy of the pudendal nerve A preliminary cadaveric study was performed on five native breed male donkeys. The pudendal nerve was 
determined among its related structures; the sacroischiatic ligament, sacrotuboral ligament, ischiatic nerve and internal pudendal blood vessels. The distance between the point of needle insertion at the most dorsal portion of the ischiorectal fossa and the superficial and deep branches of the nerve was recorded via a graded CHIBA needle. Ultrasound rectal probe was introduced via the rectum in order to obtain the proper distance and position to image the nerve and its associated blood vessels (Fig. 1).

\section{Ultrasound-guided technique of the pudendal nerve block}

Clinical bilateral pudendal nerve block was performed on six native breed adult male donkeys. Candidate donkeys aged between 8 - 15 years-old and weighted from $170-210 \mathrm{~kg}$ body weight. All animals were kept on soft laxative food for a week prior to the experiment.

The rectum was evacuated manually at the time of the application of the procedure and the perineal region and area around the anus was washed, scrubbed and disinfected. A $0.5 \mathrm{ml}$ of $2 \%$ lidocaine $\mathrm{HCL}^{*}$ was injected subcutaneously at both sides of the rectum, at the most dorsal portion of ischiorectal fossa to desensitize the point of needle insertion.

The operator introduced his left hand with the rectal probe through the rectum with its transducer directed ventrolateral. Ultrasound machine ${ }^{* *}$ was used to locate the internal pudendal blood vessels with the aid of color coded doppler ultrasound. The operator used his right hand to insert and introduce a $20-\mathrm{cm}, 18$ gauge CHIBA needle (Gallini, S.R.L, Italy) at the most dorsal portion of the ischiorectal fossa. The needle shaft could be imaged on the ultrasound screen. The needle was advanced slowly to above the level of the internal pudendal blood vessels under direct vision. The rectal probe was rotated slowly up and down to configure the both; the site of the blood vessels and the needle. A mixture of $5 \mathrm{ml}$ of lidocaine HCL and $1 \mathrm{ml}$ of methylene blue solution was injected to locate the injection site (fig. 2).

*(Depocaine 2\%, El-Depiky Pharm., Egypt).

** (Exago, France, 5 - $10 \mathrm{MHz}$ rectal probe).

Clinical and necropsy evaluation of the pudendal nerve block

Evaluation process was performed on two levels. The first was to assess the successfulness of the nerve block, and the second was to locate the dye at the injection site. Onset of loss of sensation was detected by applying needle pricks to the anus, perineum and downward to the penis and glans penis every five minutes (Fig. 4 A, B, C). The onset of anesthesia that lies between the time of injection and desensitization was recorded. The duration of anesthesia was also recorded for each donkey.

All studied animals were euthanized for educational purposes at the same day of the experiment.
Anatomical dissection of the pelvic region was performed to assess the accuracy of the injection by locating the stained segment of the nerve with the methylene blue solution. A stained segment of $2 \mathrm{~cm}$ or more of the pudendal nerve considered as an evidence of proper blockade (Campoy et al., 2008) (fig. 4D).

\section{RESULTS}

The present study revealed that the pudendal nerve fibers at the gluteal region descend caudoventrally on the medial aspect of the sacroischiatic ligament. The nerve is bounded laterally by the middle gluteus and biceps femoris muscles. The nerve passes nearly parallel to the ischiatic nerve for about $5-6 \mathrm{~cm}$, where it appears superficially and uncovered by the sacroischiatic ligament and bifurcates into superficial and deep branches. At this point, the nerve is closely related to the internal pudendal blood vessels. The deep branch divides into superficial perineal nerve that innervates the perineal region and deep genital nerve that ends at the penis. The deep branch is closely associated with the blood vessels of the penis.

The insertion point of the anesthetic needle was determined. The proper insertion point was the most dorsal aspect of the ischiorectal fossa, so that the needle can directed cranially and slightly ventral to reach the injection site about $8 \mathrm{~cm}$ deep to the insertion point. Pudendal nerve block was performed successfully in all six studied male donkeys and resulted in the desired clinical anesthetic effect. After 5.8 minutes - (mean average) of bilateral injections of the local anesthetic, the perineal region was totally desensitized. After 8 minutes - (mean average), the penis was relaxed and totally desensitized within about five minutes. Pin prick of the penile body and glans penis revealed complete desensitization and loss of pain reflexes. The glans penis and penis were examined and evaluated for presence of lesions while the animals were in a steady standing position. Lidocaine local anesthetic agent was used for standard effect. Ten $\mathrm{ml}$ of lidocaine solution for each side was sufficient and resulted in a successful block.

Several attempts were made in each animal to rotate the rectal probe slowly with the transducers plastic nose directed lateral and ventrolateral to capture the internal pudendal blood vessels. Rectal transducer with a frequency ranging from $5-8.5 \mathrm{MHz}$ was adequate to reveal the blood flow within the internal pudendal blood vessels. The time needed to perform unilateral block was 4 minutes - (mean average).

The mean average time to produce clinical anesthesia was 5.25 minutes. The mean average clinical anesthesia duration was 47.1 minutes. Penis protrusion time was 1.5 hour - (mean average) and all animals gained normal penile position.

One donkey showed severe straining and blowing from the rectum during ultrasound rectal probe introduction. 

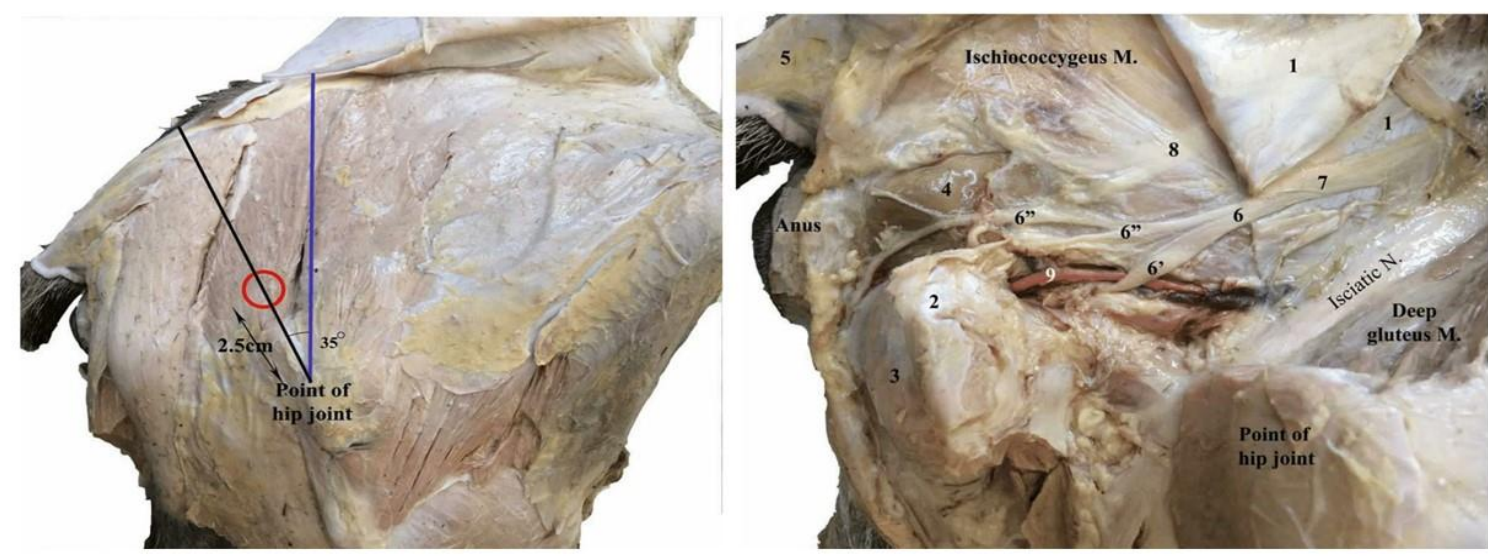

Fig. 1. Dissection of the pelvis showing the pudendal nerve and its relations. Sacroischiatic ligament (1), Ischial tuberosity (2), Ischiocavernosus M. (3), Retractor ani M. (4), Root of tail (5), Deep branch of Pudendal N. branches (6, 6', 6", 6"'), Pudendal N. (7), Caudal rectal N. (8), internal pudendal blood vessels (9).
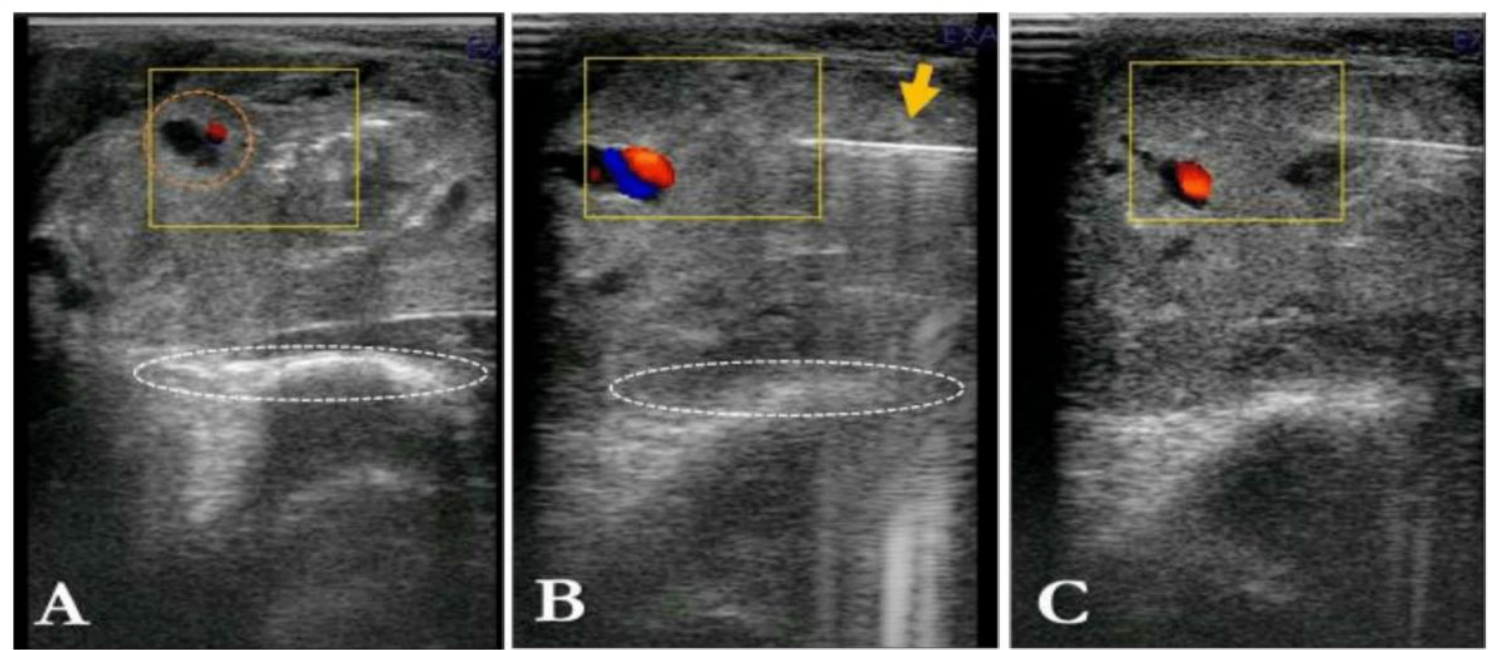

Fig. 2. Color flow doppler guided pudendal nerve block. [A] Identification of gluteal blood vessels (orange dotted circle),

ischium (white dotted circle), [B] advancing the needle above the level of internal pudendal blood vessels (yellow arrow), [C] injection of $10 \mathrm{ml}$ lidocaine solution at the level of gluteal blood vessels.
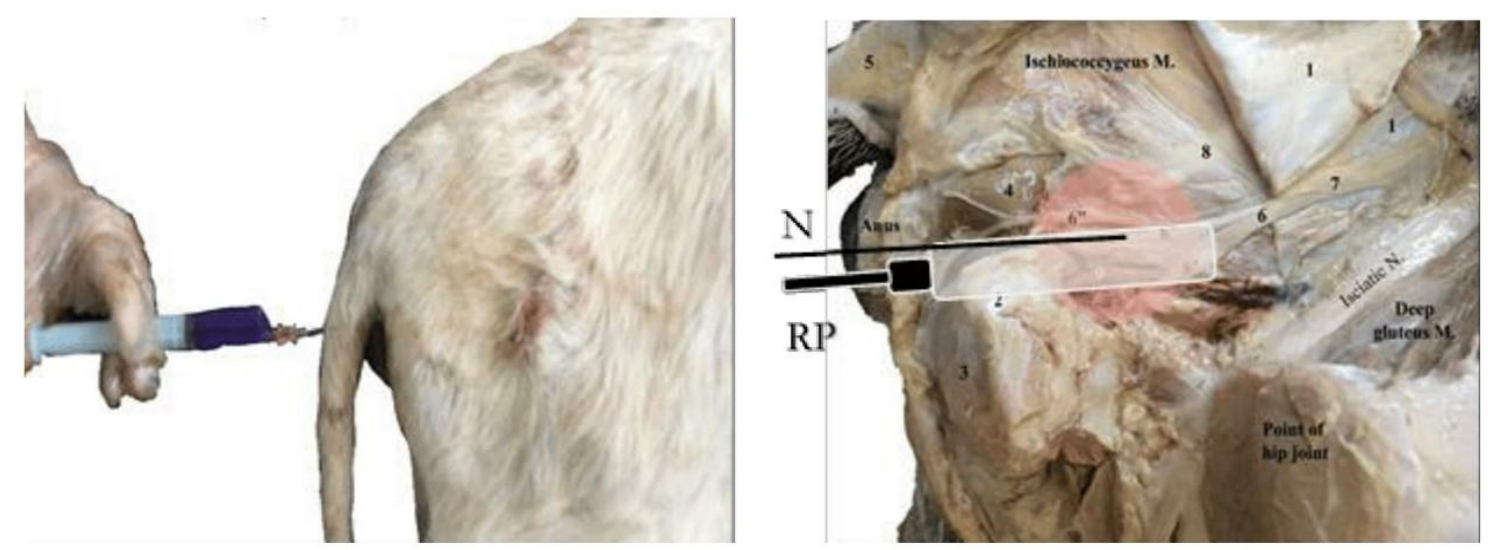

Fig. 3. Injection process. $(N)$ injection needle, $(\mathrm{RP})$ rectal probe, site of injection (the faint red circle). 

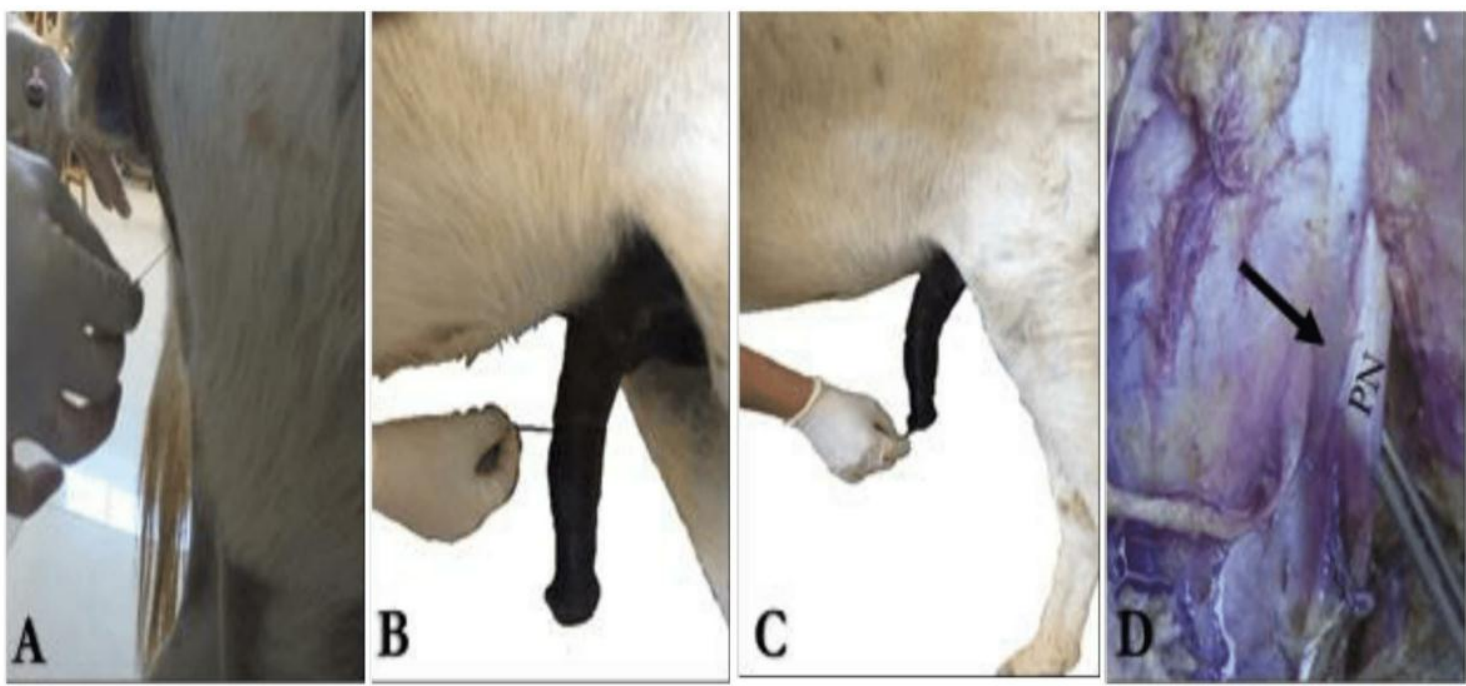

Fig. 4. Assessment process. Desensitization of perineum (A), penile body (B), glans penis (C). The pudendal nerve (PN) is

stained with the dye on dissection.

\section{DISCUSSION}

To our knowledge and according to available literature, this is the first description of a technique for blocking the pudendal nerve in donkeys using color flow doppler ultrasound. A blind pudendal nerve block technique in horses was described by Schumacher et al. (1985). This technique depends on locating the pudendal nerve through identification of the foremen of the caudal gluteal blood vessels via manual rectal palpation. The technique was difficult to achieve in donkeys because of the small size of the anus than in horses which makes rectal palpation absolutely difficult. Blind insertion of the needle without guidance may lead to severe intra and postoperative complications. A recent technique was reported by Gallacher et al. (2016) which uses electro-locating device that detect anal and perineal twitches to identify the accurate site of the pudendal nerve. The present technique uses ultrasound to detect the blood vessels accompanying the pudendal nerve. Although the experimental trials presented here required variable time to identify the internal pudendal blood vessels, but further training and gaining experience will decrease the bilateral pudendal nerve blockade time. Schumacher et al. (1985) inserted the anesthesia needle at the dorsolateral aspect of the ischiorectal fossa and advanced the needle to the rectally palpable foremen and the local anesthetic deposited to desensitize the pudendal nerve midway of its course. Gallacher et al. (2016) changed the point of needle insertion to the ventrolateral portion of the ischiorectal fossa in order to desensitize the main trunk of the pudendal nerve. In the present study, it was most appropriate to insert the anesthetic needle at the dorsolateral aspect of the ischiorectal fossa. The main target to identify was the internal pudendal blood vessels, the pudendal nerve lies about $1 \mathrm{~cm}$ dorsal to the pudendal vessels, so, it was more convenient to advance the needle from the dorsolateral aspect not to miss the anatomical landmarks and to avoid accidental injury of pudendal vessels. The results of the former study revealed that the pudendal nerve trunk is too small to be identified by ultrasound (Adami et al., 2013). In our limited $(n=6)$ experimental study, color flow doppler ultrasound machine with transrectal transducer was used to locate the internal pudendal blood vessels. First attempts were hard and required more time to introduce operator hand with the rectal probe into the small diameter anus of experimental donkeys. Further attempts were successful and required less time. The direction of the transducers' nose was recognized. Some of the potential complications during the procedure of pudendal nerve block are puncture of the rectum or, puncture of vessels with possible hematoma formation. There were no complications during or after the anesthesia procedure except for one animal. Straining and discomfort during the first attempts to introduce operators hand into the rectum was detected. This, reduced after introduction of the lubricated hand. Further repeated attempts should be avoided to reduce post-operative complications. No post-anesthesia complications recorded except for one animal that showed pneumo-rectum and rectal plowing which may be due to the anesthetic effect of anal sphincter and its relaxation. Regarding the possibility of monitoring needle advancement through the tissues, and observing the vessels and other anatomical structures, ultrasonographical guidance is likely to greatly decrease the risk of complications compared with the blind technique, and also offers the advantage of a more accurate and specific nerve block. Further studies might be beneficial to investigate the use of ultrasound guidance to monitor the pudendal nerve block in female donkeys and horses either experimentally or in clinical cases. 


\section{CONCLUSION}

This study suggests that pudendal nerve block under ultrasonographical guidance in standing male donkeys is feasible, effective and could reduce the complications associated with the blind technique. Administration of $10 \mathrm{ml}$ of lidocaine anesthetic solution for each side was readily effective and produced the desired anesthetic effect. Pudendal nerve block under ultrasound guidance is a valuable choice rather than epidural or general anesthesia for standing examination and operative procedures. Future trials will be needed to assess the usefulness and the applicability of this technique in the clinical cases.

\section{REFERENCES}

Adami, C.; Angeli, G.; Haenssgen, K.; Stoffel, M.H. and Spadavecchia, C. (2013): Development of an ultrasound-guided technique for pudendal nerve block in cat cadavers. Journal of Feline Medicine and Surgery 15, 901-907.

Budras, K.D.; Sack, W.O. and Rock, S. (2012): Anatomy of the horse, 6th ed. Schlütersche Verlagsgesellschaft $\mathrm{mbH}$ and Co. KG., HansBöckler-Alle 7, pp. 86-87.

Burnham, S.L. (2002): Anatomical differences of the donkey and mule. Proc. Am. Ass. equine Practnrs. 48, 102-109.

Campoy, L.; Martin-Flores, M.; Looney, A.L.; Erb, H.N.; Ludders, J.W.; Stewart, J.E.; Gleed, R.D. and Asakawa, M. (2008): Distribution of a lidocaine-methylene blue solution staining in brachial plexus, lumbar plexus and sciatic nerve blocks in the dog. Veterinary Anaesthesia and Analgesia 35, 348-354.

Deshmkh, S.E. and Deshpande, K.S. (1980): Internal pudendal nerve block in cows. Indian Vet. J. 57: 73-75.

Gallacher, K.; Santos, L.; Campoy, L.; Bezuidenhout, A. and Gilbert, R. (2016): Development of a peripheral nerve stimulator-guided technique for equine pudendal nerve blockade. The Veterinary Journal 217, 72-77.

Ghoshal, N.G. (1975): Spinal Nerves. In: R. Getty (Ed.) Sisson and Grossman's The Anatomy of the Domestic Animals. WB Saunders, Philadelphia, Pp: 686-687.

Johnston, G.M.; Eastment, J.K.; Wood, J.L.N. and Taylor, P.M. (2002): The confidential enquiry into perioperative equine fatalities (CEPEF): Mortality results of Phases 1 and 2. Veterinary Anaesthesia and Analgesia 29, 159-170.

Natalini, C.C. and Driessen, B. (2007): Epidural and spinal anesthesia and analgesia in the equine. Clinical Techniques in Equine Practice 6, 145153.

Olbrich, V.H. and Mosing, M. (2003): A comparison of the analgesic effects of caudal epidural methadone and lidocaine in the horse. Veterinary Anaesthesia and Analgesia 30, $156-164$.

Schumacher, J.; Bratton, G.R. and Williams, J.W. (1985): Pudendal and caudal rectal nerve blocks in the horse-An anesthetic procedure for reproductive surgery. Theriogenology 24 , $457-464$.

\section{التخدير الموضعي للعصب الفرجي في ذكور الحمير والاسترشاد بالموجات فوق الصوتية والدوبلر الملون

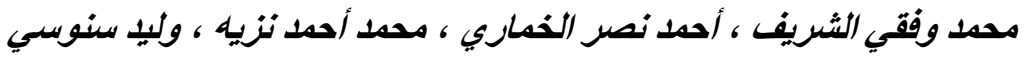

E-mail: drmwt@hotmail.com Assiut University web-site: www.aun.edu.eg

\footnotetext{
قد يحتاج اجر اء بعض العمليات الجراحية في منطقة العجان و القلفة والقضيب في ذكور الفصيلة الخيلية الي وضع الحيوان تحت تأتئير

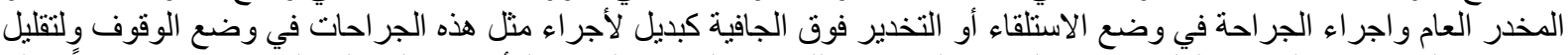

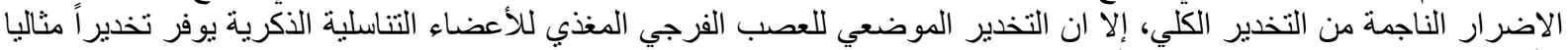

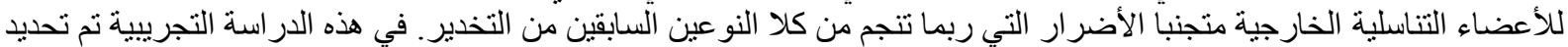

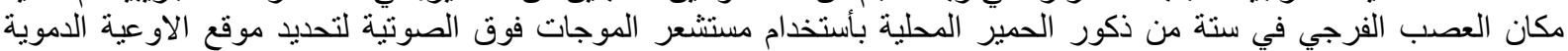

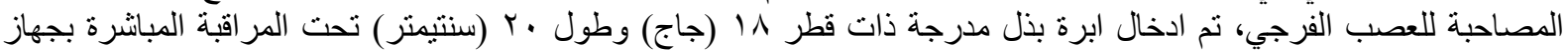

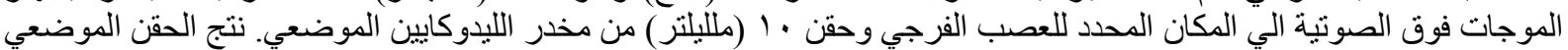

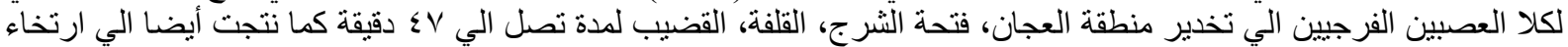

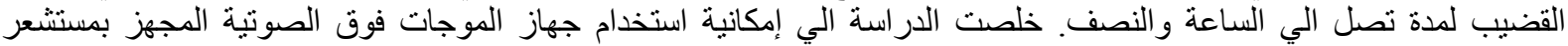

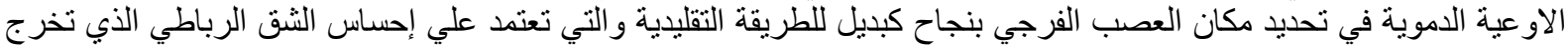

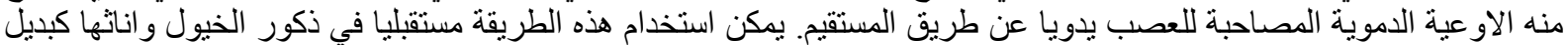
بسيط ومتاح لأجراء العطليات الجر احية والتوليد.
} 